\title{
Ferritin heavy subunit enhances apoptosis of non-small cell lung cancer cells through modulation of miR-125b/p53 axis
}

\author{
Flavia Biamonte ${ }^{1,2}$, Anna Martina Battaglia ${ }^{1,2}$, Fabiana Zolea ${ }^{1,2}$, Duarte Mendes Oliveira $\mathbb{D}^{2,3}$, llenia Aversa $a^{1,2}$, \\ Gianluca Santamaria $\mathbb{B D}^{1,3}$, Emilia Dora Giovannone ${ }^{2,3}$, Gaetano Rocco ${ }^{4}$, Giuseppe Viglietto ${ }^{2,3}$ and \\ Francesco Costanzo $1,2,3$
}

\begin{abstract}
Ferritin is a nanocage protein composed by the variable assembly of 24 heavy and light subunits. As major intracellular iron storage protein, ferritin has been studied for many years in the context of iron metabolism. However, recent evidences have highlighted its role, in particular that of the heavy subunit (FHC), in pathways related to cancer development and progression, such as cell proliferation, growth suppressor evasion, cell death inhibition, and angiogenesis. At least partly, the involvement in these pathways is due to the ability of FHC to control the expression of a repertoire of oncogenes and oncomiRNAs. Moreover, the existence of a feedback loop between FHC and the tumor suppressor p53 has been demonstrated in different cell types. Here, we show that ectopic over-expression of FHC induces the promoter hypermethylation and the down-regulation of miR-125b that, in turn, enhances p53 protein expression in non-small cell lung cancer (NSCLC) cell lines. Notably, analysis by absolute quantitative RT-PCR of FHC, miR-125b, and p53 strongly suggests that this axis might be active in human NSCLC tissue specimens. In vitro, FHC over-expression attenuates survival of NSCLC cells by inducing p53-mediated intrinsic apoptosis that is partially abrogated upon miR-125b re-expression. Overall, our findings demonstrate that FHC acts as a tumor suppressor gene, thus providing a potential molecular strategy for induction of NSCLC apoptotic cell death.
\end{abstract}

\section{Introduction}

Lung cancer is the leading cause of cancer mortality worldwide. It is a complex and heterogeneous group of diseases among which the non-small cell lung carcinoma (NSCLC) accounts for approximately $75-85 \%$ of all cases $^{1,2}$. The complex molecular pathogenesis of NSCLC involves the activation of growth-promoting proteins (KRAS, MEK-1, EGFR, BRAF, etc.) as well as the inhibition of tumor suppressor genes (p53, PTEN, etc. $)^{3,4}$.

\footnotetext{
Correspondence: Flavia Biamonte (flavia.biamonte.fb@gmail.com)

${ }^{1}$ Research Center of Biochemistry and Advanced Molecular Biology, Department of Experimental and Clinical Medicine, "Magna Græcia" University of Catanzaro, Campus Salvatore Venuta -Viale Europa, 88100 Catanzaro, Italy ${ }^{2}$ Department of Experimental and Clinical Medicine, University Magna Graecia of Catanzaro, Campus Salvatore Venuta -Viale Europa, 88100 Catanzaro, Italy Full list of author information is available at the end of the article. Edited by A. Oberst
}

Particularly, the tumor suppressor protein p53 is frequently deleted or mutated in a large number of lung cancer cell lines thus leading to the deregulation of its downstream pathways ${ }^{4}$.

As recently described, p53 activities that impact on tumor suppression extend far beyond its role in promoting cell cycle arrest or apoptosis and include also the capacity to modulate glucose and lipid metabolism as well as the redox status of the cell ${ }^{5-8}$; consequently, p53 is now considered a central hub of multiple biochemical pathways essential for the cellular homeostasis and survival. As there are many pathways downstream of p53, so they are those upstream, being the tumor suppressor activated by a variety of stress signals including irradiation, DNA damage, oncogene expression, nutrient deprivation, and hypoxia ${ }^{9,10}$.

\section{(c) The Author(s) 2018}

(c) (i) Open Access This article is licensed under a Creative Commons Attribution 4.0 International License, which permits use, sharing, adaptation, distribution and reproduction in any medium or format, as long as you give appropriate credit to the original author(s) and the source, provide a link to the Creative Commons license, and indicate if changes were made. The images or other third party material in this article are included in the article's Creative Commons license, unless indicated otherwise in a credit line to the material. If material is not included in the article's Creative Commons license and your intended use is not permitted by statutory regulation or exceeds the permitted use, you will need to obtain permission directly from the copyright holder. To view a copy of this license, visit http://creativecommons.org/licenses/by/4.0/. 
When the cell is exposed to damaging events, p53 activation is elicited by its stabilization, leading to increased intracellular amounts, and/or by the activation of the protein through a variety of post-translational modifications ${ }^{11}$. Recently, it has been reported that miRNAs are engaged in the activation pathway of p53 and its related networks at different levels through direct repression of the tumor suppressor or by the modulation of its regulators within the cell. miR-125b, miR-98, miR-150, miR214 , and miR-19b fall between the p53 direct modulators, since they directly seed-match sequences in the 3 '-UTR of the tumor suppressor mRNA. On the contrary, miR-192/ 194/215, miR-143/145, miR-29b, miR-25, miR-32, and miR-18b indirectly affect p53 through the modulation of its regulators MDM2 and MDM $4^{12-14}$.

p53 levels and/or activity are also affected by alterations of the intracellular redox state ${ }^{15-17}$. In response to cellular stress, an increased production of ROS induces p53 activation that, in turn, modulates the transcription of antioxidant target genes in order to prevent the propagation of the cell damage ${ }^{15-17}$. Ferritin plays a central role in the regulation of the intracellular redox homeostasis, due to its capacity to store iron atoms in a non-toxic form ${ }^{18}$. The variable assembly of 24 subunits of heavy (FHC) and light (FTL) types composes the protein shell provided of a central cavity in which iron is stored. The ferritin heavy subunit (FHC) plays a central role in this process since it is provided of a ferroxidase activity ${ }^{19}$. At the same time, data are mounting that FHC is involved in many pathways related to neoplastic transformation, as recently reviewed by Min Pang and Connor ${ }^{20}$; consequently, FHC is currently described as a bi-functional molecule involved in iron-related and iron unrelated biochemical routes. In many of these pathways p53 plays a central role and a tight relationship between p53 activity and FHC expression has been demonstrated ${ }^{21-23}$. p53 increases FHC expression at the translational level through the modulation of the IRP1-IRE translation regulatory system, while the over-expression of the tumor suppressor negatively modulates FHC transcription ${ }^{22}$. Conversely, FHC physically binds p53 and stabilizes the protein level under oxidative stress conditions ${ }^{23}$.

We have recently demonstrated that the intracellular increase of FHC amounts leads to enhancement of p53 protein expression in $\mathrm{H} 460$ non-small-cell lung cancer (NSCLC) cells and that this is accompanied by a significant reduction of the cell proliferation rate ${ }^{24}$. However, whether this FHC function is cell-specific or more ubiquitously effective as well as whether this function is directly mediated by perturbation of the redox metabolism or by iron-independent mechanisms are still to be explored.

In the present study we demonstrate the existence of a new regulatory axis through which FHC, regardless of its redox-related activity, enhances p53 expression by downregulation of miR-125b steady-state amounts in A549, H460, SW1573, and LXF-289 NSCLC cells lines. The model has been also validated in human clinical specimens from NSCLC patients. Moreover, we found that FHC over-expression triggered a p53-dependent apoptotic cell death in vitro.

\section{Results \\ miR-125b regulates p53 expression and activity in A549 and $\mathrm{H} 460$ cell lines}

miR-125b negatively regulates $\mathrm{p} 53$ protein expression in human neuroblastoma and lung fibroblast cells ${ }^{25}$. To obtain experimental evidences supporting p 53 as a target for miR-125b also in NSCLC cells, A549 and H460 cells were transfected with either a miR-125b mimic or antagomiR. After $48 \mathrm{~h}$ the expression of $\mathrm{p} 53$ protein was analyzed by western blot. The results shown in Fig. 1, representative of four independent transfections, demonstrated that miR-125b mimic caused at least $70 \%$ decrease of p53 protein in both cell lines $(P<0.05$; Fig. 1a); by contrast, treatment with miR-125b antagomiR resulted in at least two-fold increase in p53 protein $(P<$ 0.05; Fig. 1b). These experiments confirmed a suppressive role for miR-125b on the tumor suppressor p53 expression also in NSCLC cells. Next, we determined the effects of either miR-125b over-expression or inhibition on A549 and $\mathrm{H} 460$ cell viability. As shown in Fig. 1c both A549 and H460 cells transfected with miR-125b mimic (A549 ${ }^{\text {miR- }}$ 125 $\mathrm{b}$ mimic and $\mathrm{H} 460^{\text {miR-125 }}{ }^{\text {mimic }}$ ) showed an increase in cell growth compared with the A549 $9^{\text {cntr }}$ and $\mathrm{H} 460^{\text {cntr }}$ control cells at 24 and $48 \mathrm{~h}$. On the other hand, A549 and H460 cells treated with miR-125b antagomiR (A549 ${ }^{\text {miR- }}$ $125 \mathrm{~b}$ antagomiR and $\mathrm{H} 460^{\mathrm{miR}-125 \mathrm{~b}}$ antagomiR ) exhibited decreased cell viability when compared with cells transfected with control siRNAs (A549 $9^{\text {cntr }}$ and $\mathrm{H} 460^{\text {cntr }}$ ) (Fig. 1d).

\section{FHC epigenetically regulates miR-125b in NSCLC cells}

FHC, beside its well-described role in the control of redox balance, exerts its cancer-related functions also modulating miR-125b expression in K562 and SKOV-3 cell lines ${ }^{26,27}$. Here, we analyzed the effects of transient FHC over-expression on miR-125b steady-state amounts in NSCLC cells (A549 ${ }^{\text {pc3FHC }}$ and $\mathrm{H} 460^{\text {pc3FHC}}$ ). Results of three independent experiments showed that ectopic overexpression of FHC significantly down-regulated miR-125b of at least $60 \%$ in both cell lines $(P<0.05$; Fig. 2a).

To have an insight on FHC-mediated miR-125b regulation, we took into account that miR-125b expression is finely controlled by either an oxidative stress- or an epigenetically-dependent mechanism ${ }^{28-30}$. Thus, we measured ROS amounts in $\mathrm{A} 549^{\mathrm{pc} 3 \mathrm{FHC}}$ and $\mathrm{H} 460^{\mathrm{pc} 3 \mathrm{FHC}}$ cells compared to A549 $9^{\text {pc3DNA }}$ and $\mathrm{H} 460^{\text {pc3DNA }}$ control 


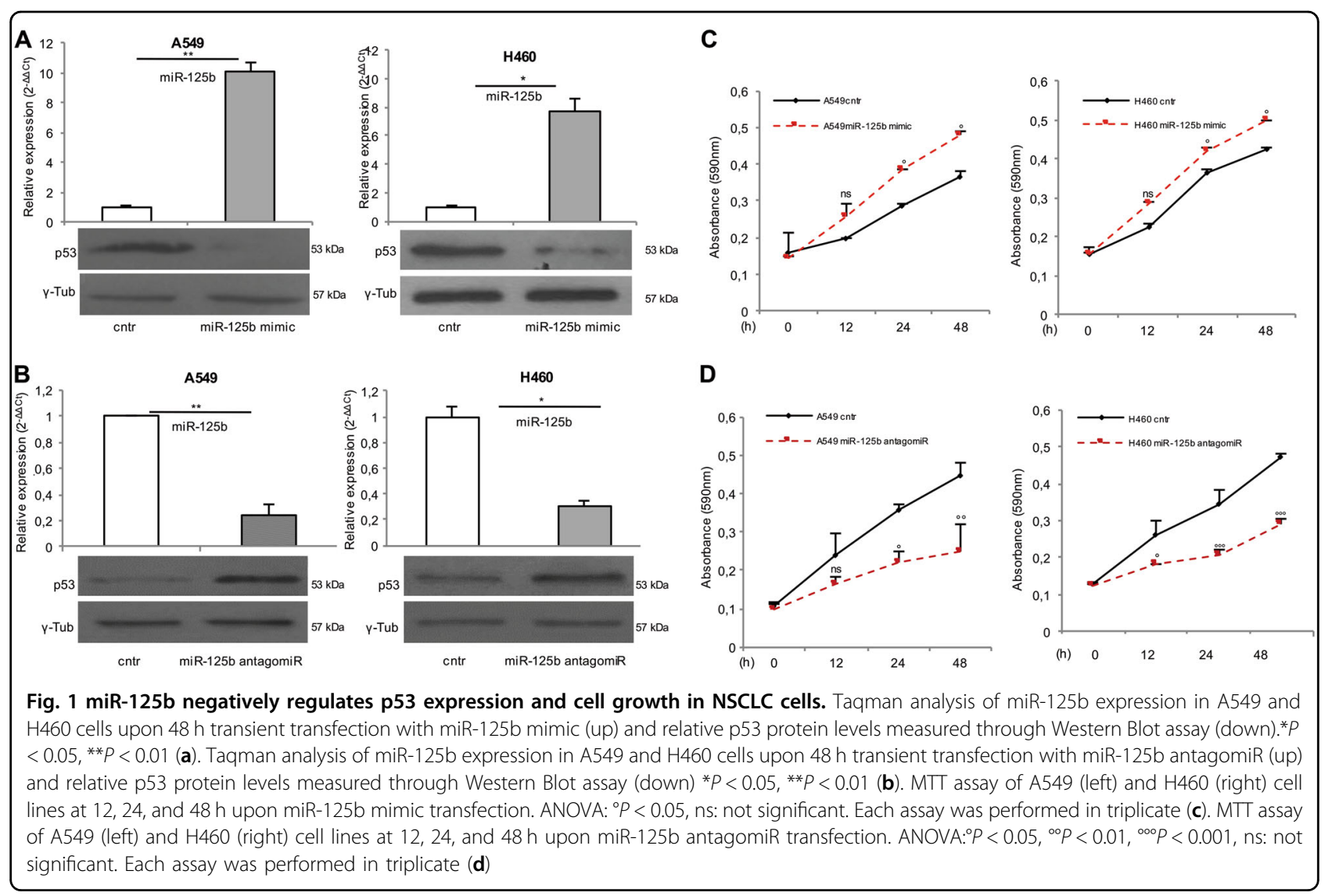

cells (Fig. S1). The absence of any significant alteration in ROS production let us to hypothesize that FHC regulated miR-125b expression through an iron/redox-independent mechanism. Next, we performed methylation-specific PCR (MSP) analysis of selected CpG islands identified within a -800 to -1020 promoter region upstream of miR-125b $b^{30}$. According to miR-125b low expression levels, this region was found to be hypermethylated in both $\mathrm{A} 549^{\mathrm{pc} 3 \mathrm{FHC}}$ and $\mathrm{H} 460^{\mathrm{pc} 3 \mathrm{FHC}}$ cells with respect to the relative control cells (Fig. 2b). A consistent up-regulation of DNA methyltransferase $1(D N M T 1)$ gene $(P<0.05$; Fig. 2c) in $\mathrm{A} 549^{\mathrm{pc} 3 \mathrm{FHC}}$ and $\mathrm{H} 460^{\mathrm{pc} 3 \mathrm{FHC}}$ cells further supported this finding. Finally, the treatment of $\mathrm{A} 549^{\mathrm{pc} 3 \mathrm{FHC}}$ and $\mathrm{H}_{460^{\mathrm{pc} 3 \mathrm{FHC}}}$ cells with $10 \mu \mathrm{M}$ of the demethylating agent decitabine (5-aza-2 deoxycytidine; AZA) for $48 \mathrm{~h}$ induced the up-regulation of miR-125b in both the cell lines $(P<0.05$; Fig. $2 \mathrm{~d})$.

\section{FHC enhances $p 53$ expression through the modulation of miR-125b in NSCLC cells}

The above results indicate that, in A549 and H460 cells, miR-125b suppresses $\mathrm{p} 53$ protein expression and that FHC over-expression inhibits miR-125b. This suggests a new regulatory activity of $\mathrm{FHC}$ on p53 through the downregulation of miR-125b. To verify this model, western blot analyses of p53 were performed in the cells transfected with the $\mathrm{pc}_{3} \mathrm{FHC}$ expression vector or co-transfected with $\mathrm{pc}_{3} \mathrm{FHC}$ and miR-125b mimic. Each transfection was performed at least three times. In the FHC-overexpressing NSCLC cells (A549 $9^{\mathrm{pc} 3 \mathrm{FHC}}$ and $\mathrm{H} 460^{\mathrm{pc} 3 \mathrm{FHC}}$ ) we observed a down-regulation of miR-125b accompanied by a roughly two-fold increase of p53 protein expression; these effects were counteracted when miR-125b levels were restored in the A549 and $\mathrm{H} 460$ cells co-transfected with $\mathrm{pc}_{3} \mathrm{FHC}$ and miR-125b mimic (A549 ${ }^{\text {pc3FHC/miR-125b mimic }}$ and H460 pc3FHC/miR-125b mimic $^{\text {p }}(P<0.05$; Fig. 3a, b).

Moreover, the $\mathrm{FHC} / \mathrm{miR}-125 \mathrm{~b} / \mathrm{p} 53$ axis was further analyzed in 22 human tumor tissue specimens derived from NSCLC patients (patient characteristics are reported in Table 1) by quantifying the three molecules transcript amounts through absolute qPCR. As represented in the box plot in Fig. 3c, non-parametric Wilcoxon signed-rank test revealed a very significant negative correlation between the expression of $\mathrm{p} 53$ and miR-125b $(P=4.503 \mathrm{e}-$ $09)$ and between FHC and miR-125b $(P=3.716 \mathrm{e}-12)$. Instead, the Wilcoxon signed-rank test returned a not significant $p$-value between p53 and FHC (Fig. 3c).

Collectively, these results support a so far uncovered model in which FHC regulates p53 through its negative control on miR-125b 

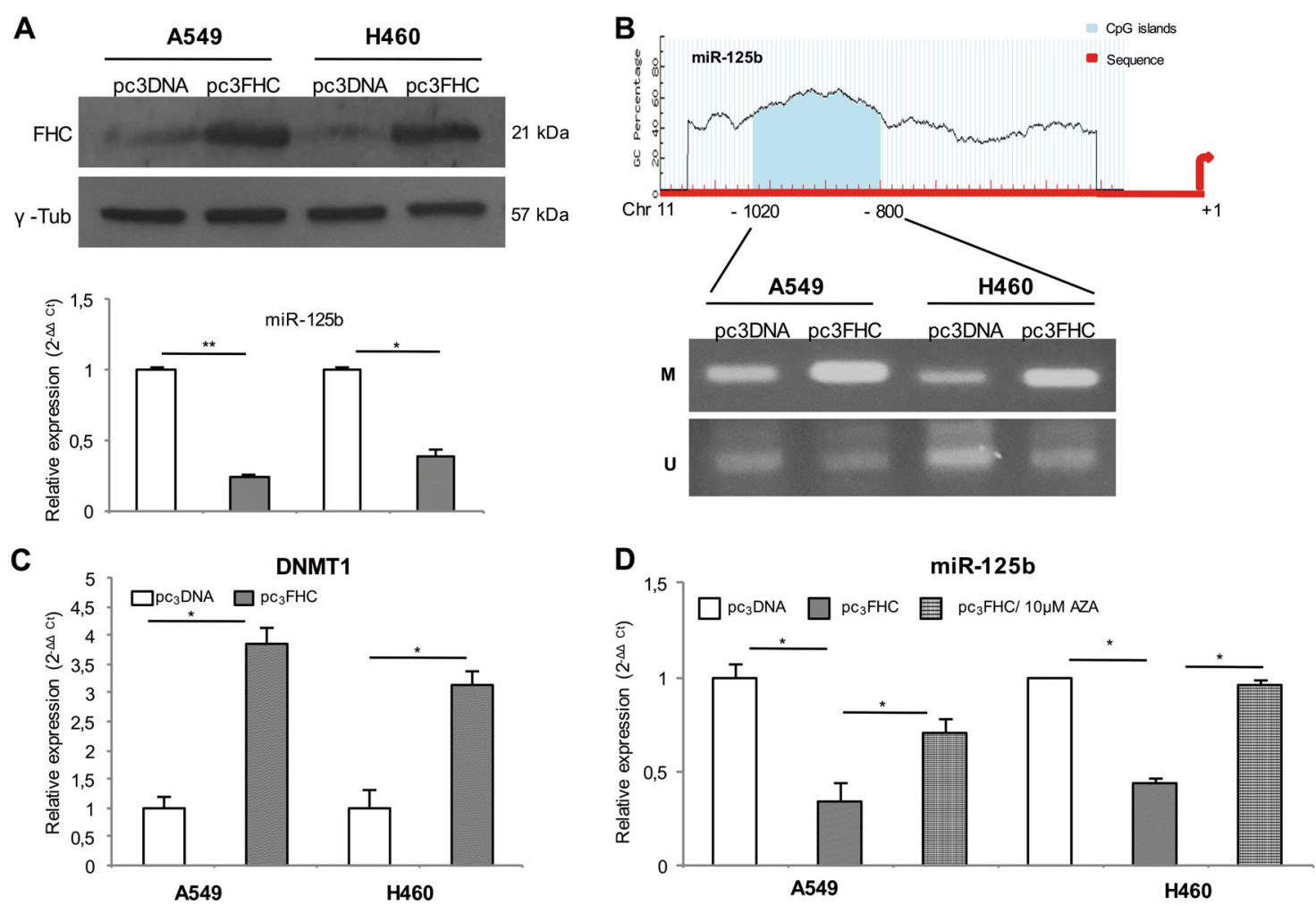

Fig. 2 FHC suppresses miR-125b expression through the hypermethylation of its promoter in NSCLC cells. Representative western blot analysis of FHC protein levels in $\mathrm{A} 549$ and $\mathrm{H} 460$ cells transfected with either the control pC 3 DNA expression vector (A549 ${ }^{\mathrm{pc} 3 \mathrm{DNA}}$ and $\mathrm{H} 460^{\mathrm{pc} 3 \mathrm{DNA}}$ ) or the FHC-specific $\mathrm{PC}_{3} \mathrm{FHC}$ expression vector ( $\mathrm{A} 549^{\mathrm{pC} 3 \mathrm{FHC}}$ and $\mathrm{H} 460^{\mathrm{pC} 3 \mathrm{FHC}}$ ) for $48 \mathrm{~h}$ (up). Taqman analysis of miR-125b expression in $\mathrm{A} 549^{\mathrm{pc} 3 \mathrm{DNA}}$, $\mathrm{A} 549^{\mathrm{pC} 3 \mathrm{FHC}}, \mathrm{H} 460^{\mathrm{pc} 3 \mathrm{DNA}}$, and $\mathrm{H} 46 \mathrm{O}^{\mathrm{PC} 3 \mathrm{FHC}}$ cells (down). Each experiment was performed on three independent biological replicates. ${ }^{*} P<0.05$, ${ }^{*} * P<$ 0.01 (a). Analysis of methylation status of a CpG site within the miR-125b gene promoter (up) through methylation-specific PCR (MSP) in A549 $9^{\text {pc3DNA }}$, A549 ${ }^{\text {pC } 3 F H C}, H 460^{\text {pc3DNA }}$, and ${\mathrm{H} 460^{\text {pC } 3 F H C}}_{\text {cells }}$ (down). Representative image of three independent biological replicates. M methylated, $U$ unmethylated (b). Real-time PCR analysis of DNMT1 expression in A549 ${ }^{\text {pC3DNA }}, A 549^{\text {pC3FHC }}, H 460^{\text {pC3DNA}}$, and H460 ${ }^{\text {pC3FHC }}$ cells; the DNMT1 expression level was significantly enhanced in $\mathrm{A} 549^{\mathrm{PC} 3 \mathrm{FHC}}$ and $\mathrm{H} 460^{\mathrm{pC} 3 \mathrm{FHC}}$ cells compared to their relative controls $\mathrm{A} 549^{\mathrm{pc} 3 \mathrm{DNA}}$ and $\mathrm{H} 460^{\mathrm{pC} 3 \mathrm{DNA}}$ cells. The assay was performed in triplicate. ${ }^{*} P<0.05$ (c). Taqman analysis of miR-125b expression in A549 and $\mathrm{H} 460$ control cells, and $\mathrm{A} 549^{\mathrm{PC} 3 \mathrm{FHC}}$ and $\mathrm{H} 460^{\mathrm{PC} F \mathrm{FHC}} \mathrm{Cells}$ upon treatment with $10 \mu \mathrm{M}$ 5-aza-2 deoxycytidine (AZA) for $48 \mathrm{~h}$. The assay was performed in triplicate. ${ }^{*} P<0.05$ (d)

$\mathrm{FHC} / \mathrm{miR}-125 \mathrm{~b} / \mathrm{p} 53$ axis affects $\mathrm{A} 549$ and $\mathrm{H} 460$ cell survival by modulating the intrinsic apoptotic pathway

To assess how the FHC/miR-125b/p53 axis impacted on NSCLC cell behavior, we carried out the cytofluorimetric cell cycle and apoptosis assays. Representative graphs from PI flow cytometry analyses demonstrate that FHC ectopic over-expression did not alter A549 and H460 cell distribution within the major phases of the cell cycle (Fig. S2). Conversely, representative plots and quantifications of Annexin V/7-AAD apoptosis assays highlighted that the percentage of apoptosis, considered as the sum of early apoptosis (Annexin $\mathrm{V}^{+} / 7-\mathrm{AAD}^{-}$) and late apoptosis (Annexin $\mathrm{V}^{+} / 7-\mathrm{AAD}^{+}$), was remarkably increased in A549 and H460 FHC-overexpressing cells compared with the relative controls (A549 ${ }^{\mathrm{pc} 3 \mathrm{FHC}}$ vs A549 ${ }^{\mathrm{pc} 3 \mathrm{DNA}}: 70.6 \% \pm$ 3.4 vs $9.1 \% \pm 1.9, P<0.01)\left(\mathrm{H}_{4} 60^{\mathrm{pc} 3 \mathrm{FHC}}\right.$ vs $\mathrm{H} 460^{\mathrm{pc} 3 \mathrm{DNA}}$ : $60.2 \% \pm 8.4$ vs $5.8 \% \pm 0.8, P<0.01$ ) (Fig. $4 \mathrm{a}, \mathrm{b})$. In addition, the mimic-mediated miR-125b rescue in A549 $9^{\mathrm{pc} 3 \mathrm{FHC} / \mathrm{miR}-}$ 125b mimic and $\mathrm{H} 460^{\mathrm{pc} 3 \mathrm{FHC} / \mathrm{miR}-125 \mathrm{~b} \text { mimic }}$ was able to partially counteract the pro-apoptotic effect of the sole $\mathrm{H}$ -

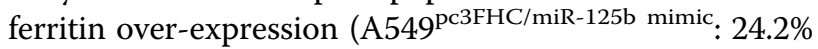
$\pm 1.05, P<0.05)\left(\mathrm{H} 460^{\mathrm{pc} 3 \mathrm{FHC} / \mathrm{miR}-125 \mathrm{~b} \text { mimic }}: 34.9 \% \pm 5.1, P\right.$ $<0.05$ ) (Fig. 4a, b).

Moreover, western blot analyses showed an increase in $\mathrm{BAX} / \mathrm{Bcl}-2$ ratio, indicative of the activation of the intrinsic apoptotic pathway within A549 $9^{\mathrm{Pc} 3 \mathrm{FHC}}$ and $\mathrm{H} 460^{\text {pc3FHC }}$ cells, further confirmed by the increased caspase-9 cleavage (Fig. 4c). Otherwise, western blot analysis of FAS and caspase- 8 cleavage suggested that extrinsic apoptotic pathway was not involved (Fig. S3).

\section{FHC/miR-125b/p53 axis modulates apoptosis in SW1573 and LXF-289 NSCLC cells}

To evaluate the generalizability of $\mathrm{FHC} / \mathrm{miR}-125 \mathrm{~b} / \mathrm{p} 53$ axis in NSCLC cells, the amounts of miR-125b and p53 were determined in SW1573 and LXF-289 cells upon 

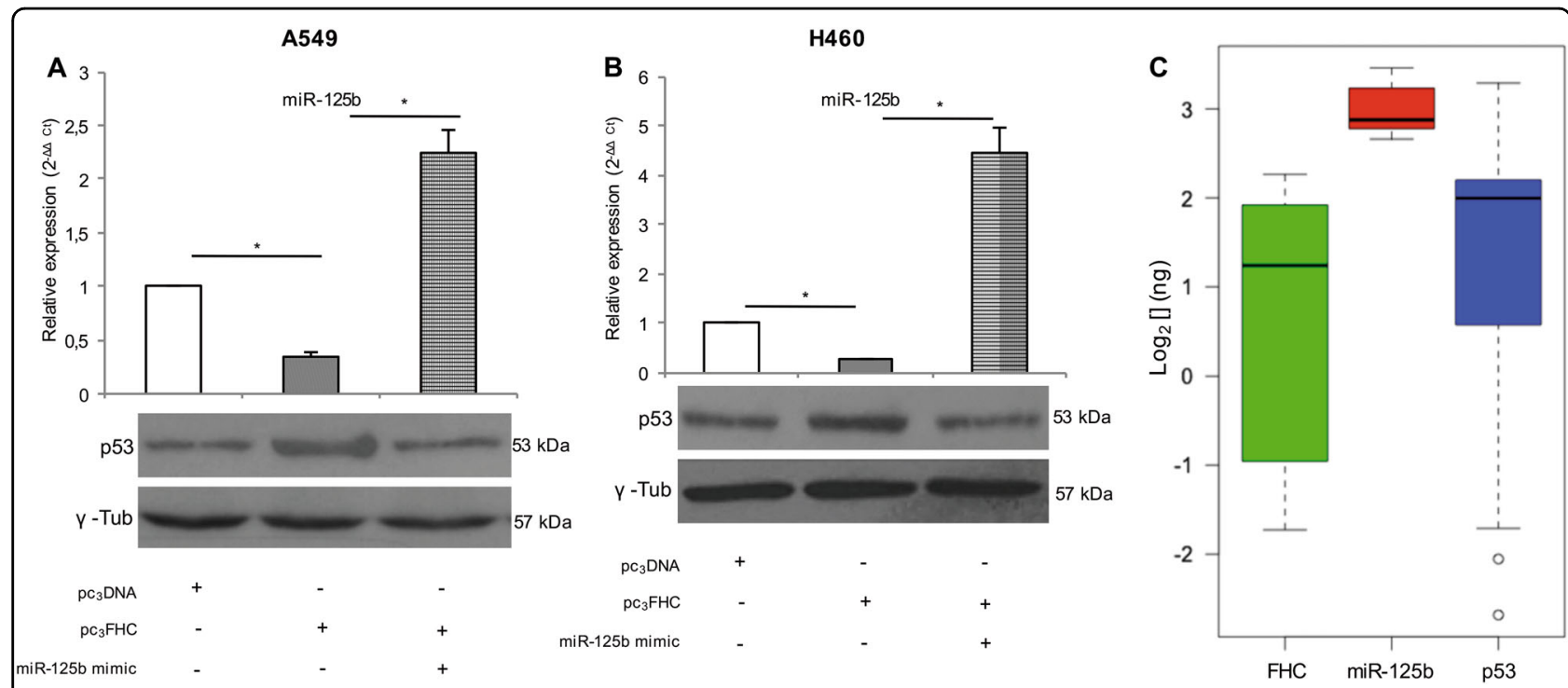

Fig. 3 FHC enhances p53 expression through the negative modulation of miR-125b in NSCLC in vitro and ex vivo. Taqman analysis of miR$125 \mathrm{~b}$ expression in $\mathrm{A} 549$ and $\mathrm{H} 460$ cells either transfected with $\mathrm{pC}_{3} \mathrm{FHC}$ expression vector alone ( $\mathrm{A} 549^{\mathrm{pC} 3 \mathrm{FHC}}$ and $\mathrm{H} 460^{\mathrm{pC} 3 \mathrm{FHC}}$ ) or CO-transfected with

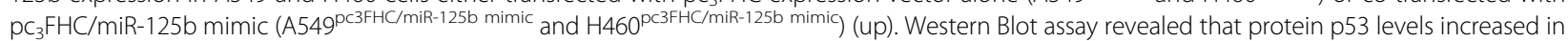

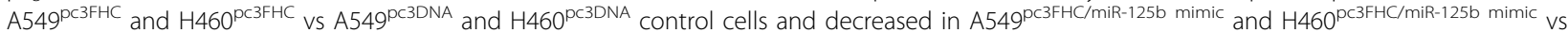

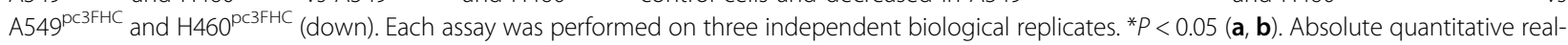
time PCR of miR-125b, p53, and FHC in 22 human NSCLC tissue specimens are reported as Box Plot. Absolute qPCR was performed on three independent technical replicates. Wilcoxon signed-rank test ${ }^{* * *} P<0.001 p 53$ vs miR-125b, Wilcoxon signed-rank test ${ }^{000} p<0.001 F H C$ vs miR-125b (c)

FHC over-expression. Results confirmed that the increase in FHC amounts is accompanied by miR-125b downregulation that, in turn, enhances p53 levels in both the cell lines (Fig. S4a). We further explored the functional effects of p53 modulation on the apoptotic rate of both SW1573 and LXF-289 cells. As reported in the representative plots in Fig. S4b, the quantification of Annexin V/PI-positive cells highlighted that FHC over-expression increased the percentage of apoptosis, considered again as the sum of both early apoptosis (Annexin $\mathrm{V}^{+} / \mathrm{PI}^{-}$) and late apoptosis (Annexin $\mathrm{V}^{+} / \mathrm{PI}^{+}$): SW1573 ${ }^{\mathrm{pc} 3 \mathrm{FHC}}$ vs SW1573 ${ }^{\text {pc3DNA }}: 35.1 \% \pm 2.1$ vs $\left.14.1 \% \pm 0.3, P<0.05\right)$ $\left(\mathrm{LXF}-289^{\mathrm{Pc} 3 \mathrm{FHC}}\right.$ vs LXF-289 ${ }^{\text {Pc3DNA }}: 15.0 \% \pm 0.4$ vs $5.1 \% \pm$ $0.3, P<0.05)$.

\section{Discussion}

Numerous evidences link intracellular iron metabolism and tumorigenesis ${ }^{31,32}$; among them, a segment puts directly in relation iron and tumor suppressor p53 functions. Indeed, it has been demonstrated that iron depletion up-regulates p53 at post-transcriptional level ${ }^{33}$, as well as that heme is able to bind p53 and to down-regulate its activity, by modifying localization and stability ${ }^{34}$. p53 is able, for its part, to modulate the intracellular iron homeostasis: this observation stems from the discovery that ISCU (iron-sulfur cluster assembly enzyme) belongs to the family of p53 target genes, being provided with an intronic p53-binding site. ISCU, in turn, controls, at post- transcriptional level, the expression of FHC and transferrin receptor (TFRC), two key proteins of iron metabolism; in particular, by acting on the IRP1/IRE regulatory system, ISCU positively modulates FHC translation and negatively modulates TFRC mRNA half-life ${ }^{35}$.

The links between p53 and FHC do not end with the ISCU post-transcriptional role: upon oxidative stress, FHC might physically interact with p53 and increase its transcriptional activity ${ }^{23}$. The interaction and the downstream p53 activation also takes place with a mutant form of FHC devoid of ferroxidase activity, strongly suggesting that the binding capability is iron-independent. On the other hand, p53, following its over-expression, is recruited by the NF-Y transcription factor onto the FHC promoter, where it acts as a strong negative regulator of ferritin transcriptional efficiency ${ }^{22}$. Finally, it has been shown that $\mathrm{H}$-chain rich acidic isoferritins, released from hepatocytes in vitro, up-regulate p53 and mediate apoptotic processes through a mechanism which again appears to be unrelated to the ferritin role in balancing the intracellular iron pool $^{36}$.

The major finding of our work is the identification of a novel and so far uncovered relationship between FHC and p53 in NSCLC cells through miR-125b. The set of our results indicate that $\mathrm{FHC}$ is the upstream molecule of this regulatory axis by virtue of its ability to modulate miR$125 \mathrm{~b}$ amounts; the microRNA, for its parts, controls the expression and therefore the activity of p53 (Fig. 5). 


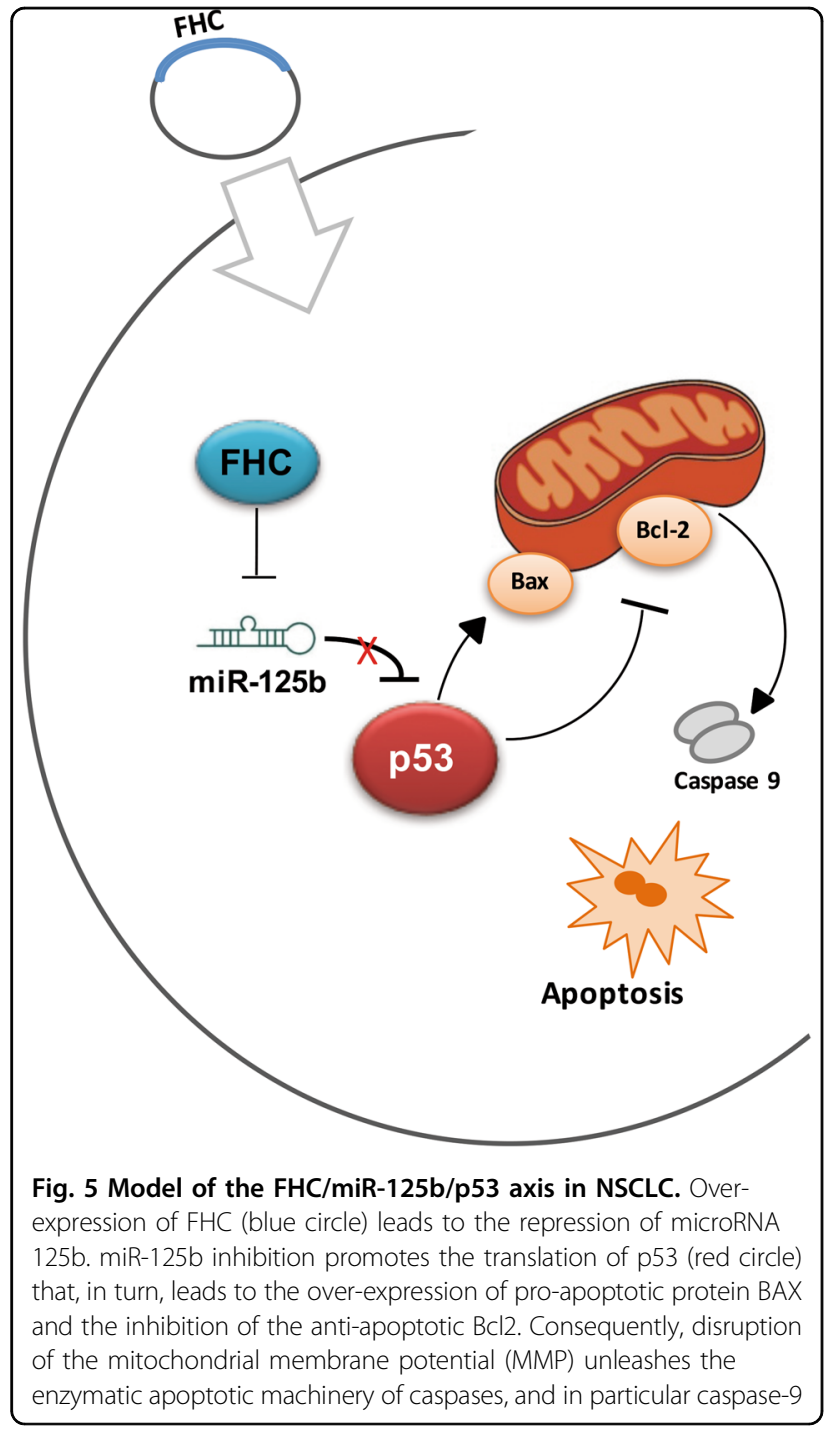

Analyses of the molecular mechanisms underlying this regulatory axis strongly suggest that FHC exerts its effects on miR-125b/p53 regardless of the iron/redox status of the cell being ROS production unaltered upon FHC overexpression (Fig. S1).

On the contrary, several observations indicate that FHC epigenetically suppresses miR-125b expression: (i) FHC over-expression is accompanied by the hypermethylation of a previously computationally mapped $\mathrm{CpG}$ island in miR-125b promoter region ${ }^{30}$; (ii) $\mathrm{FHC}$ over-expression is accompanied by the up-regulation of DNMT1 gene expression, and (iii) miR-125b expression is partially restored after treatment of $\mathrm{FHC}$-overexpressing cells with the demethylating agent 5-Aza for $48 \mathrm{~h}$. The observation that DNMT1 steady-state amounts are induced by FHC over-expression deserves further and more detailed investigations. DNMT1 has been described as a direct target of a repertoire of miRNAs including miR-126 $6^{37,38}$.
We already found that miR-126 belongs to the FHCdependent miRNAs in K562 erythroleukemia cells ${ }^{26}$. Thus, we determined miR-126 levels in A549, H460, SW1573, LXF-289 control, and FHC-overexpressing cells. Results show that miR-126 is down-regulated, at variable extents, in the FHC-overexpressing cells (A549 ${ }^{\mathrm{pc} 3 \mathrm{FHC}}$, H460 ${ }^{\text {pc3FHC }}$, SW1573 ${ }^{\text {pc3FHC }}$, and LXF-289 ${ }^{\text {pc3FHC }}$ ) where DNMT1 is up-regulated (data not shown).

p53 is a target of miR-125b in human neuroblastoma cells and in lung fibroblasts ${ }^{25}$; here, by using miR-125b mimic and antagomiR we demonstrate that this control also takes place in NSCLC cells. Consequently, miR-125b, through its suppressive activity on p53, acts as oncomiRNA in NSCLC cells; accordingly, cells transfected with miR-125b antagomiR show a consistent growth decrease. Moreover, the miRNA intermediate position between FHC and p53 is confirmed by recovery experiments in which a specific mimic is used to restore miR125b levels suppressed upon FHC over-expression. By absolute RT-qPCR analysis we also determined the steady-state amounts of $F H C$, miR-125b, and p53 in 22 NCSLC tissue samples. Wilcoxon signed-rank test highlights that the levels of $F H C$ and miR-125b are inversely related, as are those of miR-125b and p53, with a high statistical significance; this strongly suggests that the relationship of FHC with p53 through miR-125b might be also active in vivo. Further analyses on a large size of clinical samples as well as in mouse models are required to confirm these findings.

p53 activity as a tumor suppressor depends on its ability in regulating downstream pathways involved in DNA repair, senescence, cell-cycle arrest, and/or apoptosis. Depending on the extent of injury, p53 may promote cell cycle arrest to activate DNA repair mechanisms and restore homeostasis; conversely, in case of a severe injury p53 induces cells to undergo death by apoptosis ${ }^{9,10,39,40}$. In this work, we show that $\mathrm{FHC} / \mathrm{miR}$-125b-mediated upregulation of p53 forces NSCLC cells to undergo apoptosis without affecting cell cycle progression, thus suggesting that the wealth of the ferritin subunit could constitute a severe damage to the cell. Apoptosis is driven by p 53 transcriptional induction of a number of genes, including the death receptors Fas and Dr4, which in turn activate the extrinsic apoptotic pathway ${ }^{9,41}$. Furthermore, p53 may increase the transcription of death agonist genes such as BAX and, in parallel, repress the expression of death antagonist such as Bcl-2, thus inducing mitochondrial outer membrane permeabilization and the intrinsic apoptosis pathway, ${ }^{9,41}$. Here, we show that $\mathrm{FHC} / \mathrm{miR}$ 125b/p53-mediated apoptosis occurs through the activation of the intrinsic pathway as indicated by the increase of the Bax/Bcl-2 ratio and caspase- 9 activation.

As recently reviewed, the role of $\mathrm{FHC}$ in malignancies is conflicting since the ferritin subunit might function as a 


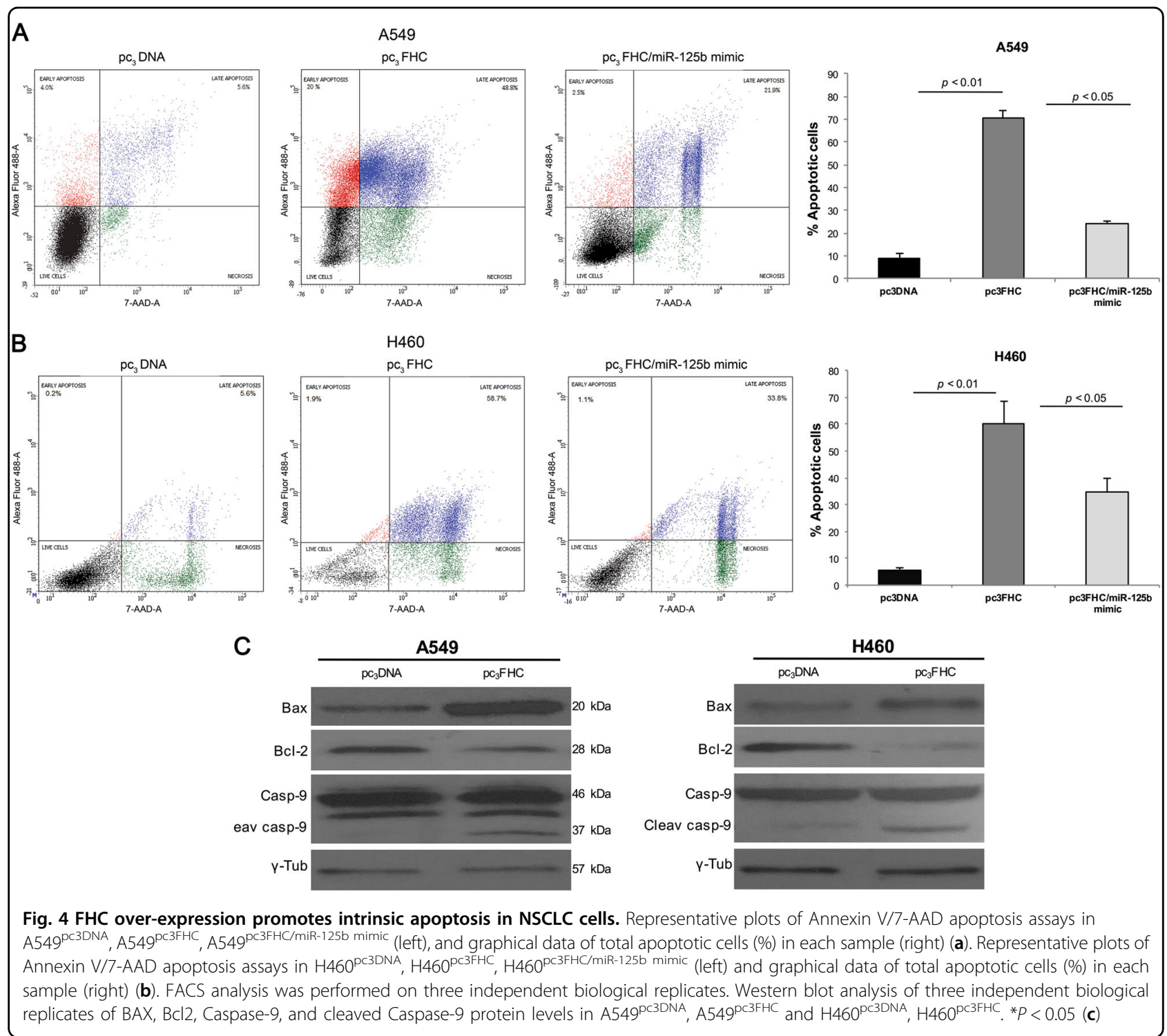

tumor suppressor or oncogene depending on the cellular context $^{20}$. The natural conclusion of our findings on NSCLC cells is that FHC acts as a tumor suppressor in these cells as it happens in breast and ovarian cancer cell lines $^{27,42}$. This role is exerted by $\mathrm{H}$-ferritin via multiple mechanisms such as the regulation of CXCL12/CXCR-4 axis in breast cancer cells or the modulation of a specific microRNA pattern in ovarian cancer cells ${ }^{27,40}$. Some of these mechanisms might be also active in NSCLC cells along with the predominant modulation of $\mathrm{p} 53$. This is suggested by the observation that a percentage of apoptotic cells still remains when the miR-125b/p53 axis is contrasted by miR-125b mimic co-transfection.

Finally, the ability of FHC over-expression in triggering p53-mediated apoptosis might have profound implications in designing new therapeutic approaches in NSCLC, also taking into account its current employment as a nanocarrier due to its unique protein architecture.

\section{Materials and methods}

Cell cultures, human tissue samples, and treatments

Human NSCLC cell lines A549, H460, SW1573, and LXF-289 were obtained from the American Type Culture Collection (ATCC, Rockville, MD, USA). All the cell lines were maintained in RPMI640 media (Sigma-Aldrich, St. Louis, MO, USA) supplemented with $10 \%$ (v/v) fetal bovine serum (FBS) (Invitrogen, San Diego, CA) and 1\% (v/v) penicillin and streptomycin (Sigma-Aldrich, St. Louis, MO, USA), in adherent cultures at $37^{\circ} \mathrm{C}$ in a humidified $5 \%$ $\mathrm{CO}_{2}$ atmosphere. Cell lines were tested for mycoplasma contaminations and STR profiled for authentication.

Twenty-two tumor biopsies were obtained from the University Magna Graecia of Catanzaro (Catanzaro, Italy); 
Table 1 Patient characteristics

Frequency (number)

\begin{tabular}{ll}
\hline Age at diagnosis (years) & \\
$<50$ & $5 \%(1)$ \\
$50-59$ & $14 \%(3)$ \\
$60-69$ & $31 \%(7)$ \\
$>70$ & $50 \%(11)$ \\
Histotype & \\
Adenocarcinoma & $32 \%(7)$ \\
Squamous cell carcinoma & $23 \%(5)$ \\
Large cell carcinoma & $9 \%(2)$ \\
Not otherwise specified (NOS) & $36 \%(8)$ \\
Grade & \\
I & 0 \\
II & $55 \%(12)$ \\
III/IV & $31 \%(7)$ \\
Unknown & $14 \%(3)$ \\
Staging & \\
I & $9 \%(2)$ \\
II & $36 \%(8)$ \\
III & $9 \%(2)$ \\
IV & $5 \%(1)$ \\
\hline
\end{tabular}

patient accrual was conducted according to the Internal Review Board of the INT Fondazione Pascale (Naples, Italy) (CEI:556,10 of 12/3/2010). The study was approved by the Internal Review Board of Mater Domini/University Magna Graecia (Catanzaro, Italy), in the meeting of 16/03/2011.

Twenty-two cases of NSCLC patient specimens were frozen and stored in liquid nitrogen until use. The characteristics of patients included are described in Table 1.

For 5-aza-2'-deoxycytidine (AZA) treatment, A549 ${ }^{\text {pc3FHC }}$, and $\mathrm{H}_{460^{\mathrm{pc} 3 \mathrm{FHC}}}$ were seeded at $5 \times 10^{5}$ per well in six-well plates and cultured with $10 \mu \mathrm{M}$ AZA (Sigma-Aldrich) for $48 \mathrm{~h}$, respectively. The medium containing drug was replaced every $24 \mathrm{~h}$. RNA was isolated and qRT-PCR was carried out to evaluate the restoration of miR-125b after AZA treatment.

In vitro transfection of A549 and $\mathrm{H} 460$ cells with synthetic miR-125b antagomiR or mimic

Synthetic miR-125b antagomiR or mimic were purchased from Thermo Fisher Scientific (Waltham, Massachusetts, USA). A549 and H460 control cells $\left(5 \times 10^{5}\right)$ were seeded in a six-well plate (Corning Incorporated, Corning, NY, USA). After $24 \mathrm{~h}$, cells were transfected with $50 \mathrm{nM}$ of hsa-miR-125b-5p mirVana miRNA mimic (A549 ${ }^{\text {miR-125b mimic }}$ and H460 ${ }^{\text {miR-125b mimic }}$ ) or $50 \mathrm{nM}$ of has-miR-125b-5p mirVana miRNA antagomiR (A549 ${ }^{\mathrm{miR}-}$ 125b antagomiR and $\mathrm{H} 460^{\mathrm{miR}-125 \mathrm{~b} \text { antagomiR }}$ ) using Lipofectamine 2000 reagents according to the manufacturer's instructions (Thermo Fisher Scientific, Waltham, Massachusetts, USA). Fifty nanomolar of mirVana mimic or antagomiR-negative controls were also used. After $6 \mathrm{~h}$ of incubation, the transfection medium was replaced with fresh RPMI1640 medium. Then, after $48 \mathrm{~h}$ of transfection, cells were centrifuged and total RNA was extracted from A549 ${ }^{\text {miR-125 mimic }}$ and $\mathrm{H} 460^{\text {miR-125b mimic }}$, A $549^{\text {miR-125b antagomiR }}$ and $H 460^{\text {miR-125b antagomiR }}$, A549 ${ }^{\text {cntr }}$ and $\mathrm{H} 460^{\mathrm{cntr}}$.

\section{FHC transient over-expression in NSCLC cells}

A549, H460, SW1573, and LXF-289 cells were plated into six-well plates at $5 \times 10^{5}$ cells/well and grown overnight prior to transfection. $F H C$ transient over-expression was performed by using a specific $\mathrm{pc}_{3} \mathrm{FHC}$ expression vector (A549 ${ }^{\mathrm{pc} 3 \mathrm{FHC}}, \mathrm{H} 460^{\mathrm{pc} 3 \mathrm{FHC}}, \mathrm{SW} 1573^{\mathrm{pc} 3 \mathrm{FHC}}$ and LXF$289^{\mathrm{pc} 3 \mathrm{FHC}}$ ) and $\mathrm{pc}_{3} \mathrm{DNA}$ as negative control (A549 ${ }^{\mathrm{pc} 3 \mathrm{DNA}}$, $\mathrm{H} 460^{\text {pc3DNA }}$ SW1573 ${ }^{\text {pc3DNA}}$, and LXF-289 ${ }^{\text {pc3DNA }}$ ) as previously reported in Zolea et al. $^{24}$. All transfections were performed three times using the Lipofectamine 2000 reagents according to the manufacturer's recommendations (Thermo Fisher Scientific, Waltham, Massachusetts, USA). After 48 h, FHC-specific over-expression was checked at protein levels through western blot. Data concerning FHC over-expression are reported as mean of three independent biological replicates.

\section{RNA isolation and qRT-PCR analysis}

Total RNA isolation, single-stranded complementary DNA (cDNA) generation and relative-quantitative RTPCR were performed as previously reported in Zolea et al. $^{24}$. Validated qRT-PCR primers for DNMT1 were from Thermo Fisher Scientific. For miRNA quantification we used a TaqMan MicroRNA assay kit (Thermo Fisher Scientific, Waltham, Massachusetts, USA) and specific primer sets for U6 snRNA (Assay ID: 001973) and mature hsa-miR-125b-5p (Assay ID: 000449) (Thermo Fisher Scientific, Waltham, Massachusetts, USA) according to the manufacturer's instructions. GAPDH and U6 snRNA were used as internal normalizers for mRNA and miRNA, respectively.

Absolute qRT-PCR was used to determine the expression of $F H C$, p53, and miR-125b in 22 tumor tissue specimens. Briefly, starting from a sample of known template concentration, a 5-point 10-fold serial standard curve was prepared, and the concentration of all other samples was calculated by simple interpolation of each threshold cycle 
(Ct) into this standard curve. Gene expression data are reported as $\log _{2}$ [] (ng) and represent the mean of three independent technical replicates.

\section{Western blotting}

Total cell lysates were prepared using RIPA buffer ${ }^{24,26}$. Each protein sample $(40-50 \mu \mathrm{g})$ was separated by $10-15 \%$ SDS-PAGE and then transferred to nitrocellulose membranes $^{24,26}$. Membranes were incubated with primary antibodies at $4{ }^{\circ} \mathrm{C}$ overnight. Antibodies against FHC (B12) (1:200, sc-376594), p53 (A-1) (1:500, sc-393031), BAX (B-9) (1:500, sc-7480), and Bcl-2 (100) (1:500, sc-509) were purchased from Santa Cruz Biotechnology (Santa Cruz Biotechnology, Dallas, Texas); antibodies against Caspase-9 (1:500, \#9502), Caspase-8 (1C12) (1:500, \#9746), and Fas (C18C12) (1:500, \#4233) were purchased from Cell Signaling Technology (Danvers, Massachusetts, USA). Membranes were then incubated with secondary antibodies, HRP-conjugated goat anti-mouse IgG (1:2000, sc-516102; Santa Cruz Biotechnology, Dallas, Texas) and HRP-conjugated mouse anti-rabbit IgG (1:2000, sc-2357; Santa Cruz Biotechnology, Dallas, Texas), and immunoreactive bands were visualized with the ECL western blotting detection system (Santa Cruz Biotechnology, Dallas, Texas). To ensure equal loading of proteins a goat polyclonal anti- $\gamma$-Tubulin antibody (C-20) (1:3000; sc7396; Santa Cruz Biotechnology) was used. The protein band intensity on western blots was quantified and normalized to that of $\gamma$-Tubulin by using ImageJ software (http://rsb.info.nih.gov/ij/).

\section{MTT assay}

A total of $5 \times 10^{4}$ cells/well were seeded into a 24-well plate. 3-[4,5-Dimethylthiaoly]-2,5-diphenyltetrazolium bromide (MTT) (Sigma Aldrich, St. Louis, MO, USA) assay was performed to detect proliferation of A549 and $\mathrm{H} 460$ upon transient over-expression of miR-125b (A549 ${ }^{\text {miR-125b mimic }}$ and $\mathrm{H} 460^{\text {miR-125b mimic }}$ ) or transient inhibition of miR-125b (A549 $9^{\text {miR-125b antagomiR }}$ and H460 ${ }^{\text {miR-125b antagomiR }}$ ) for 12,24 , and $48 \mathrm{~h}$. Fresh MTT $2 \mathrm{mg} / \mathrm{ml}$ (Sigma Aldrich, St. Louis, MO, USA), resuspended in phosphate-buffered saline (PBS), was added to each well. After $2 \mathrm{~h}$ incubation, culture medium was discarded and replaced with $200 \mu \mathrm{l}$ of isopropanol. Optical density was measured at $590 \mathrm{~nm}$ in a spectrophotometer. Each transfection was performed in triplicate. For each sample, MTT assay was performed in quadruplicate.

\section{ROS detection}

ROS were determined by incubating A549 $9^{\text {pc3DNA }}$, $\mathrm{H} 460^{\text {pc3DNA }}, \mathrm{A} 549^{\text {pc3FHC }}$, and $\mathrm{H} 460^{\text {pc3FHC }}$ with the redoxsensitive probe 2'-7'-DCF (CM-H2DCFDA; Thermo Fisher Scientific, Waltham, Massachusetts, USA $)^{43}$.

\section{Methylation-specific PCR}

MSP for detecting the methylation status of miR-125b promoter was performed as previously reported by Zhang et al $^{30}$. Bisulfite conversion was performed using EpiTect Bisulfite Kit Specific (Qiagen, Hilden, Germany); PCR was performed using AmpliTaq Gold ${ }^{\mathrm{ma}}$ DNA Polymerase Kit and primers as reported by Zhang et al. ${ }^{30}$.

\section{Cell cycle analysis}

A total of $2 \times 10^{6}$ cells were fixed with $100 \%$ ethanol and stored at $4{ }^{\circ} \mathrm{C}$ for overnight. Cells were rehydrated with PBS for $10 \mathrm{~min}$ at RT and then cells were stained with propidium iodide (PI) staining solution contained with $50 \mu \mathrm{g} / \mathrm{ml}$ PI (Sigma Aldrich, St. Louis, MO, USA), $100 \mu \mathrm{g} / \mathrm{ml}$ DNase-free RNase A (Calbiochem, La Jolla, CA), and $0.01 \%$ NP-40 (USB, Cleveland, OH) in PBS for $60 \mathrm{~min}$ at room temperature. Stained cells were analyzed for cell cycle analysis in BD LSRFortessa ${ }^{\mathrm{TM}} \mathrm{X}-20$ (BD Biosciences, San Jose, CA) and FlowJo software.

\section{Apoptosis analysis}

For identifying cells actively undergoing apoptosis, a double staining with Annexin $\mathrm{V}$ and PI was performed using Alexa Fluor ${ }^{\circledR} 488$ Annexin V/Dead Cell Apoptosis Kit (Thermo Fisher Scientific, Waltham, Massachusetts, USA) according to the manufacturer's instructions. After staining, cells were incubated at room temperature for $15 \mathrm{~min}$ in the dark. Each tube was diluted with $400 \mu \mathrm{l}$ of Annexin Binding Buffer and then cells were analyzed by flow cytometry using the BD LSR Fortessa ${ }^{\mathrm{TM}} \mathrm{X}-20$ (BD Biosciences, San Jose, CA) and FACS Diva 7.0 program (BD Biosciences, San Jose, CA).

\section{Statistics}

Where appropriate, data were evaluated by performing a simple comparison between two values using Student's $t$-test. With $t$-tests, we compared the mean of each group assuming that the groups consisted of independent observations with equal variances. When testing time series, the assumption of independence is usually not reasonable. Consequently, even comparing the means of two or more time series is considerably more difficult than with independent data. So, when we were interested in determining whether the means from more than two groups or time series were equal or not, we performed analysis of variance (ANOVA). Summary statistics are presented as the mean \pm S.D. A $P$-value of $<0.05$ was considered statistically significant.

Wilcoxon signed-rank test was used to determine the statistical significance of genes expression across the lung cohort of patients measured by absolute qRT-PCR. Expression values were defined as $\log _{2}$. A $P$-value of $<0.05$ was considered statistically significant. 


\section{Acknowledgements}

This work was supported from Associazione Italiana per la Ricerca sul Cancro (AIRC) to G.V. We thank Caterina Alessi for the editorial assessment.

\section{Author details}

'Research Center of Biochemistry and Advanced Molecular Biology, Department of Experimental and Clinical Medicine, "Magna Græcia" University of Catanzaro, Campus Salvatore Venuta -Viale Europa, 88100 Catanzaro, Italy. ${ }^{2}$ Department of Experimental and Clinical Medicine, University Magna Graecia of Catanzaro, Campus Salvatore Venuta -Viale Europa, 88100 Catanzaro, Italy. ${ }^{3}$ Interdepartmental Center of Services (CIS), University Magna Graecia of Catanzaro, Campus Salvatore Venuta -Viale Europa, 88100 Catanzaro, Italy. ${ }^{4}$ Department of Thoracic Surgical and Medical Oncology, Division of Thoracic Surgery, Istituto Nazionale Tumori, IRCCS, Pascale Foundation, Naples, Italy

\section{Conflict of interest}

The authors declare that they have no conflict of interest.

\section{Publisher's note}

Springer Nature remains neutral with regard to jurisdictional claims in published maps and institutional affiliations.

Supplementary Information accompanies this paper at (https://doi.org/ 10.1038/s41419-018-1216-3).

Received: 13 June 2018 Revised: 3 November 2018 Accepted: 12 November 2018

Published online: 05 December 2018

\section{References}

1. Molina, J. R., Yang, P., Cassivi, S. D., Schild, S. E. \& Adjei, A. A. Non-small cell lung cancer: epidemiology, risk factors, treatment, and survivorship. Mayo Clin. Proc 83, 584-594 (2008)

2. Zappa, C. \& Mousa, S. A. Non-small cell lung cancer: current treatment and future advances. Transl. Lung Cancer Res. 5, 288-300 (2016).

3. Cooper, W. A., Lam, D. C., O'Toole, S. A. \& Minna, J. D. Molecular biology of lung cancer. J. Thorac. Dis. 5, 479-490 (2013).

4. Gibbons, D. L., Byers, L. A. \& Kurie, J. M. Smoking, p53 mutation, and lung cancer. Mol. Cancer Res. 12, 3-13 (2014).

5. Bieging, K. T., Mello, S. S. \& Attardi, L. D. Unravelling mechanisms of p53mediated tumour suppression. Nat. Rev. Cancer 14, 359-370 (2014).

6. Vousden, K. H. \& Lane, D. P. p53 in health and disease. Nat. Rev. Mol. Cell Biol. 8 275-283 (2007)

7. Liu, D. \& Xu, Y. p53, oxidative stress, and aging. Antioxid. Redox Signal. 15 1669-1678 (2011).

8. Maillet, A. \& Pervaiz, S. Redox regulation ofp53, redox effectors regulated by p53: a subtle balance. Antioxid. Redox Signal. 16, 1285-1294 (2012).

9. Fridman, J. S. \& Lowe, S. W. Control of apoptosis by p53. Oncogene 22, 9030-9040 (2003)

10. Kastan, M. B., Canman, C. E. \& Leonard, C. J. P53, cell cycle control and apoptosis: implications for cancer. Cancer Metastasis. Rev. 14, 3-15 (1995).

11. Kruse, J. P. \& Gu, W. Modes of p53 regulation. Cell 137, 609-622 (2009).

12. Takwi, A. \& Li, Y. The p53 pathway encounters the microRNA world. Curr. Genomics 10, 194-197 (2009).

13. Feng, Z., Zhang, C., Wu, R. \& Hu, W. Tumor suppressor p53 meets microRNAs. J. Mol. Cell Biol. 3, 44-50 (2011).

14. Liu, J., Zhang, C., Zhao, Y. \& Feng, Z. MicroRNA control of p53. J. Cell Biochem. 118, 7-14 (2017).

15. Macip, S. et al. Influence of induced reactive oxygen species in p53-mediated cell fate decisions. Mol. Cell Biol. 23, 8576-8585 (2003).

16. Liu, B., Chen, Y. \& St Clair, D. K. ROS andp53: a versatile partnership. Free Radic. Biol. Med. 44, 1529-1535 (2008).
17. Montero, J., Dutta, C., van Bodegom, D., Weinstock, D. \& Letai, A. p53 regulates a non-apoptotic death induced by ROS. Cell Death Differ. 20, 146514-146574 (2013).

18. Orino, K. et al. Ferritin and the response to oxidative stress. Biochem. J. 357, 241-247 (2001).

19. Torti, F. M. \& Torti, S. V. Regulation of ferritin genes and protein. Blood 99 3505-3516 (2002)

20. Min Pang, B. S. \& Connor, J. R. Role of ferritin in cancer biology. J. Cancer Sci. Ther. 7, 155-160 (2015).

21. Zhang, F., Wang, W., Tsuji, Y., Torti, S. V. \& Torti, F. M. Post-transcriptional modulation of iron homeostasis during p53-dependent growth arrest J. Biol. Chem. 283, 33911-33918 (2008).

22. Faniello, M. C. et al. p53-mediated downregulation of $\mathrm{H}$ ferritin promoter transcriptional efficiency via NF-Y. Int. J. Biochem. Cell Biol. 40, 2110-2119 (2008).

23. Lee, J. H., Jang, $H_{\text {., }}$ Cho, E. J. \& Youn, H. D. Ferritin binds and activates p53 under oxidative stress. Biochem. Biophys. Res. Commun. 389, 399-404 (2009).

24. Zolea, F. et al. Caffeine positively modulates ferritin heavy chain expression in H460 cells: effects on cell proliferation. PLOS ONE 11, e0163078 (2016).

25. Le, M. T. et al. MicroRNA-125b is a novel negative regulator of p53. Genes Dev. 23, 862-876 (2009).

26. Biamonte, F. et al. H-ferritin-regulated microRNAs modulate gene expression in K562 cells. PLOS ONE 10, e0122105 (2015).

27. Lobello, N. et al. Ferritin heavy chain is a negative regulator of ovarian cancer stem cell expansion and epithelial to mesenchymal transition. Oncotarget 7, 62019-62033 (2016).

28. Manca, S. et al. Oxidative stress activation of miR-125b is part of the molecular switch for Hailey-Hailey disease manifestation. Exp. Dermatol. 20, 932-937 (2011).

29. Chen, H. \& Xu, Z. Hypermethylation-associated Silencing of miR-125a and miR-125b: a potential marker in colorectal cancer. Dis. Markers 2015, 345080 (2015).

30. Zhang, Y. et al. miR-125b is methylated and functions as a tumor suppressor by regulating the ETS1 proto-oncogene in human invasive breast cancer. Cancer Res. 71, 3552-3562 (2011).

31. Torti, S. V. \& Torti, F. M. Iron and cancer: more ore to be mined. Nat. Rev. Cancer 13, 342-355 (2013).

32. Zhang, C. \& Zhan, F. Iron homeostasis and tumorigenesis: molecular mechanisms and therapeutic opportunities. Protein Cell 6, 88-100 (2015)

33. Dongiovanni, P. et al. Iron-dependent regulation of MDM2 influences p53 activity and hepatic carcinogenesis. Am. J. Pathol. 176, 1006-1017 (2010).

34. Shen, J. et al. Iron metabolism regulates p53 signaling through direct hemep53 interaction and modulation of p53 localization, stability, and function. Cell Rep. 7, 180-193 (2014)

35. Funauchi, Y. et al. Regulation of iron homeostasis by the p53-ISCU pathway. Sci. Rep. 5, 16497 (2015).

36. Bresgen, $\mathrm{N}$. et al. Iron-mediated oxidative stress plays an essential role in ferritin-induced cell death. Free Radic. Biol. Med. 48, 1347-1357 (2010).

37. Ceribelli, A. et al. MicroRNAs in systemic rheumatic diseases. Arthritis Res. Ther 13, 229 (2011).

38. Shen, N., Liang, D., Tang, Y., de Vries, N. \& Tak, P. P. MicroRNAs-novel regulators of systemic lupus erythematosus pathogenesis. Nat. Rev. Rheumatol. 8 701-709 (2012).

39. Sullivan, K. D., Galbraith, M. D., Andrysik, Z. \& Espinosa, J. M. Mechanisms of transcriptional regulation by p53. Cell Death Differ. 25, 133-143 (2018).

40. Aubrey, B. J., Kelly, G. L., Janic, A., Herold, M. J. \& Strasser, A. How does p53 induce apoptosis and how does this relate to p53-mediated tumour suppression? Cell Death Differ. 25, 104-113 (2018).

41. Elmore, S. Apoptosis: a review of programmed cell death. Toxicol. Pathol. $\mathbf{3 5}$ 495-516 (2007)

42. Aversa, I. et al. Epithelial-to-mesenchymal transition in FHC-silenced cells: the role of CXCR4/CXCL12 axis. J. Exp. Clin. Cancer Res. 36, 104 (2017).

43. Zolea, $\mathrm{F}$. et al. $\mathrm{H}$ ferritin silencing induces protein misfolding in $\mathrm{K} 562$ cells: a Raman analysis. Free Radic. Biol. Med. 89, 614-623 (2015). 